\title{
2D Vessel Segmentation Using Local Adaptive Contrast Enhancement
}

\author{
Dominik Schuldhaus ${ }^{1,2}$, Martin Spiegel ${ }^{1,2,3,4}$, Thomas Redel ${ }^{3}$, \\ Maria Polyanskaya ${ }^{1,3}$, Tobias Struffert ${ }^{2}$, Joachim Hornegger ${ }^{1,4}$, Arnd Doerfler ${ }^{2}$ \\ ${ }^{1}$ Pattern Recognition Lab, University Erlangen-Nuremberg, Germany \\ ${ }^{2}$ Department of Neuroradiology, University Erlangen-Nuremberg, Germany \\ ${ }^{3}$ Siemens AG Healthcare Sector, Forchheim, Germany \\ ${ }^{4}$ Erlangen Graduate School in Advanced Optical Technologies, SAOT, Germany \\ martin.spiegel@informatik.uni-erlangen.de
}

\begin{abstract}
D vessel segmentation algorithms working on 2D digital subtraction angiography (DSA) images suffer from inhomogeneous contrast agent distributions within the vessels. In this work, we present a novel semi-automatic vessel segmentation method based on local adaptive contrast enhancement. Either a forward projected 3D centerline or a set of manual selected seed points define the vessel branches to be segmented on the image. The algorithm uses bilateral filtering followed by local contrast enhancement to eliminate intensity inhomogeneity within the vessel region that is caused by unequally distributed contrast agent. Our segmentation algorithm is extensively evaluated on 45 different DSA images and exhibits an average Hausdorff distance of 22 pixels and sensitivity of $89 \%$.
\end{abstract}

\section{Introduction}

X-ray based 2D DSA plays a major role in diagnosis, treatment planning and assessment of cerebrovascular disease, i.e. aneurysms, arteriovenous malformations and intracranial stenosis. 2D vessel segmentation is considered as important support for analyzing complex vessels, i.e. measuring vessel diameter, length or aneurysm neck and dome size. Within the literature there are various kinds of vessel detection and segmentation methods $[1,2]$ available, however, $2 \mathrm{D}$ vessel segmentation techniques applied on 2D DSA images are hardly described [3]. Fig. 1 depicts three image examples our algorithm is working with. At first glance, the segmentation of such kind of vessels seems to be easy but the local inhomogeneous contrast agent distribution (compare the areas indicated by the red and green circles on Fig. 1), patient movement as well as the smooth intensity ramp between vessels and background make it a difficult task. Global threshold segmentation will fail because it will not handle the heterogeneous intensities distributions within the vessel regions. This paper introduces a semi-automatic $2 \mathrm{D}$ vessel segmentation technique based on local adaptive contrast enhancement to equalize the intensity inhomogeneity. The algorithm is extensively evaluated using a database of 45 different DSA images. 


\section{Materials and Methods}

\subsection{Initialization and Preprocessing}

The first step within our algorithm is indicated by the selection of the vessel branches that will be segmented. This selection is done by the placement of manual seed points along the vessel branches. The manual seed points are connected using Dijkstra's algorithm [4] together with an intensity-based cost function. All centerline points $\mathbf{c}_{i} \in \mathbb{R}^{2}$ are stored within set $\mathbf{C}$ defined as

$$
\mathbf{C}=\left\{\mathbf{c}_{i}\right\}_{i=0}^{N-1}
$$

where $N$ denotes the total number of centerline points. If the corresponding 3D vessel segmentation, the $3 \mathrm{D}$ centerline and the projection geometry are available, the vessels of interest may be automatically defined by the forward projection of the $3 \mathrm{D}$ centerline onto the 2D DSA image. Fig. 2a shows a 2D DSA frame together with the centerline (red) defining the vessels of interest. After vessel selection the noise within the 2D DSA images are reduced while preserving edges. For that purpose, bilateral filtering [5] is applied.

\subsection{Local Adaptive Contrast Enhancement}

This section describes the core part of our algorithm, i.e. the local adaptive contrast enhancement to properly handle the inhomogeneous distribution of the contrast agent within the vessel region. The idea is to place small overlaying boxes along the vessel centerline $\mathbf{C}$ and perform an intensity mapping of the pixel intensities within each box taking the lower pixel value for overlaying regions. Fig. 3a illustrates schematically the box alignment around a vessel branch. The hessian matrix [6] is computed at each centerline point (orange line) to get the two eigenvectors and eigenvalues which define the orientation of the box (blue arrows). The eigenvector pointing across the vessel is used to estimate

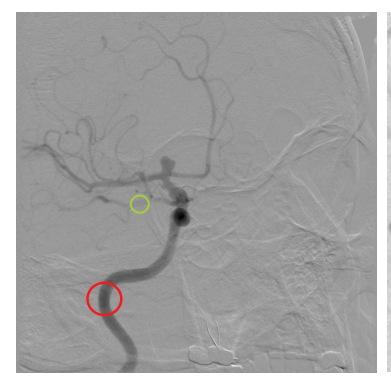

(a) $S_{10}$

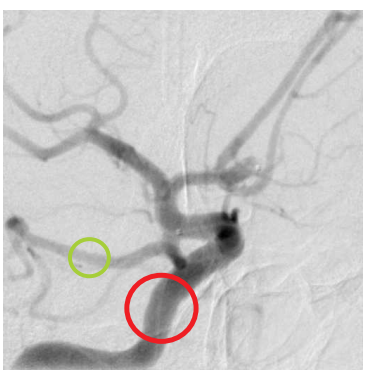

(b) $S_{17}$

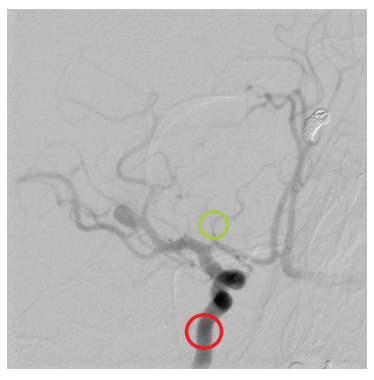

(c) $S_{22}$

Fig. 1. Three different 2D DSA images. Green and red circles indicate the inhomogeneous contrast agent distribution. $S_{i}$ abbreviates the study number within our evaluation database. 
the vessel diameter by a 1-D intensity profile. Hence, the box length across the vessel is the vessel diameter plus a certain offset such that the box certainly covers the underlying vessel branch. The length of the box in vessel direction is kept constant. Each box is now associated with one sigmoid function $f$ that is applied to perform the contrast enhancement by intensity mapping. $f$ is defined as follows

$$
f(I)=\frac{1}{1+e^{-\left(\frac{I-\beta}{\alpha}\right)}} \quad \alpha, \beta \in \mathbb{R},
$$

where $I$ is the intensity before the mapping, $\alpha$ denotes the slope and $\beta$ the translation of the sigmoid function. In our case, $\alpha$ is kept constant and $\beta$ is adapted according to the intensity mean of each box. Fig. 3b depicts three different boxes before and after contrast enhancement. The result of the local adaptive contrast enhancement is shown in Fig. 2b.

\subsection{Binary Threshold and Skeleton-Based Noise Reduction}

A binary threshold filter is applied on the contrast enhanced image Fig. $2 \mathrm{~b}$ to get a binary mask as illustrated in Fig. 2c. Since our contrast enhancement is based on boxes, small isolated artifacts appear in the vicinity of the vessels. To remove these artifacts the binary image is skeletonized by a medial axis based skeletonization approach. Each skeleton part has to exhibit a minimum number

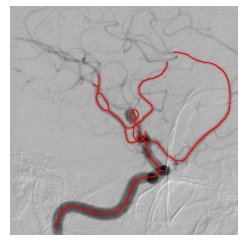

(a)

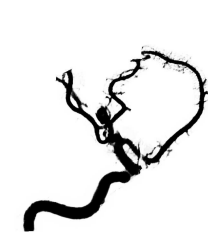

(b)

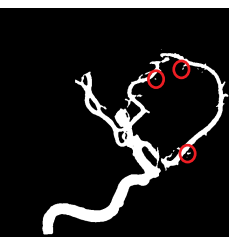

(c)

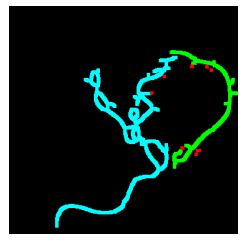

(d)

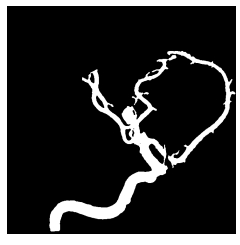

(e)

Fig. 2. Example steps of the image processing pipeline. (a) initialization via centerline, (b) local adaptive contrast enhanced image [5\% of pixels black, $79 \%$ white], (c) binary thresholding including artifacts, (d) skeleton image and (e) final segmentation result.

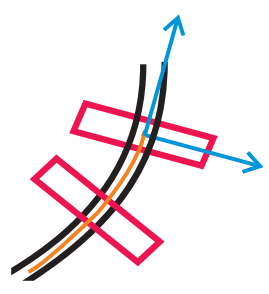

(a)

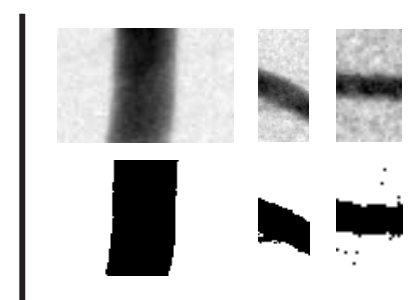

(b)

Fig. 3. (a) Illustration of box parameters; (b) the upper row depicts cutouts of a 2D DSA frame and the lower row shows the result after local adaptive contrast enhancement. 
of points which was defined heuristically. All skeleton parts below this number (see red points in Fig. 2d) together with its artifacts are removed. The final segmentation result is shown in Fig. 2e.

\section{$3 \quad$ Results}

The algorithm was extensively evaluated using a database of 45 studies $\left(\left\{S_{i}\right\}_{i=1}^{45}\right)$. The dimension varied between $512 \times 512$ and $1440 \times 1440$. The pixel spacing in $x / y$ was $0.154 / 0.154 \mathrm{~mm}$ for $S_{1}$ to $S_{18}$ and $0.308 / 0.308 \mathrm{~mm}$ for $S_{19}$ to $S_{45}$. For each study a gold standard segmentation was built by a neuroradiologist. The algorithm achieved an average Hausdorff distance of 22 pixels with a standard deviation of 6.0 and an average sensitivity of $89 \%$ with a standard deviation of 0.04. The Hausdorff distance was computed by the euclidean distance transform on the segmentation result. Fig. 4 summarizes all quantitative measurements and Fig. 5 shows three segmentation results. The left column depicts the 2D DSA images, whereas the right column shows the corresponding gold standard segmentations (red) and the segmentation results (gray) as an overlay.

\section{Discussion}

Our local adaptive contrast enhanced vessel segmentation algorithm has shown that it properly handles the intensity variation within vessel regions to perform a smooth segmentation using threshold image filtering. The extensive evaluation demonstrates that our approach is clinical applicable and able to come up with quantitative measurements during diagnosis and treatment planning. As an outlook, the 2D vessel segmentation results may be used as ground truth to overlay and validate 3D vessel segmentation results based on 3D rotational angiography images.
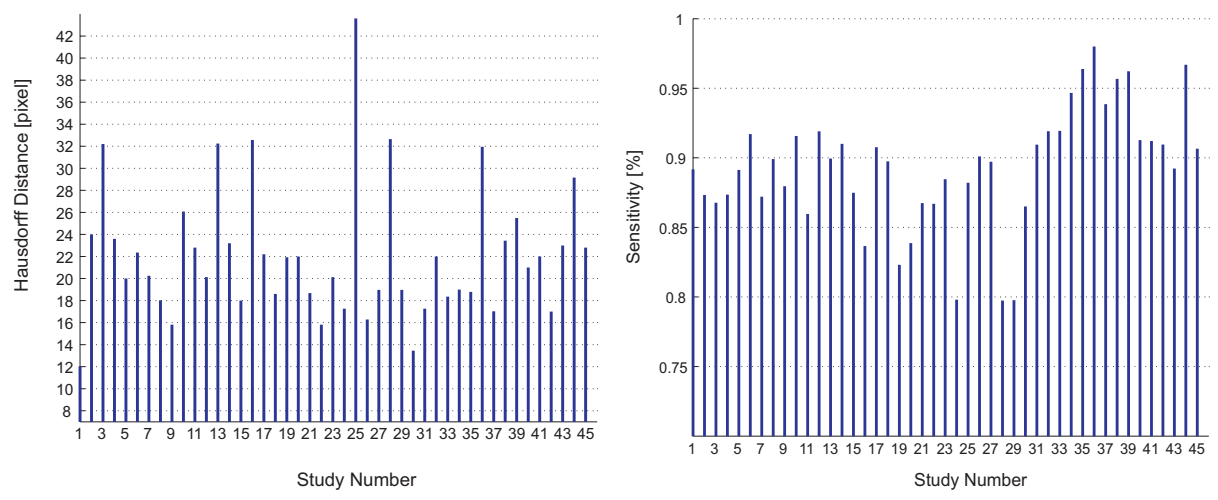

Fig. 4. Overview about the evaluation results, i.e. Hausdorff distance and sensitivity. 
Fig. 5. Segmentation results for study $S_{7}, S_{9}$ and $S_{20}$. Left column depicts the original 2D DSA images. Right column shows the corresponding gold standard segmentations (red) and our segmentation results (gray) as overlay. The red line represents the centerline (set $\mathrm{C}$ ) defining the vessels of interest.

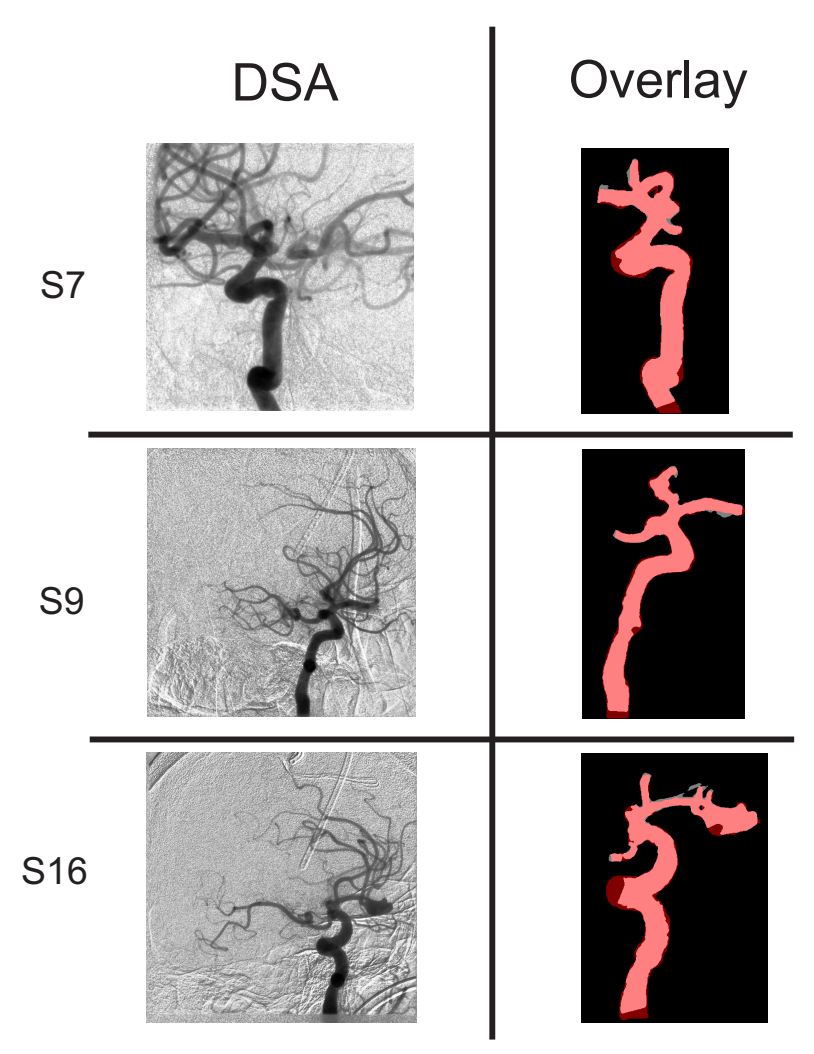

\section{References}

1. Lesage D, Angelini E, Bloch I, et al. A review of 3D vessel lumen segmentation techniques: models, features and extraction schemes. Med Image Anal. 2009;13(6):81945 .

2. Kirbas C, Quek F. A review of vessel extraction techniques and algorithms. ACM Compute Surv. 2004;36(2):81-121.

3. Sang N, Li H, Peng W, et al. Knowledge-based adaptive thresholding segmentation of digital subtraction angiography images. Image Vis Comput. 2007;25(8):1263-70.

4. Dijkstra EW. A note on two problems in connexion with graphs. Numerische Mathematik. 1959;1:269-71.

5. Tomasi C, Manduchi R. Bilateral Filtering for Gray and Color Images. Proc ICCV. 1998; p. 839-846.

6. Frangi A, Niessen W, Vincken K, et al. Multiscale vessel enhancement filtering. In: Medical Image Computing and Computer-Assisted Interventation. Boston, USA: Springer-Verlag; 1998. p. 130-37. 